\title{
Levantamento parasitológico de moluscos limnicos encontrados no município de São
}

\section{Cristóvão, Sergipe, Brasil}

Parasitological survey of limnic molluscs found in the municipality of São Cristóvão, Sergipe, Brazil

Relevamiento parasitológico de moluscos límbicos encontrados en el municipio de São Cristóvão, Sergipe, Brasil

Sara Antônia Silva da Vitória ORCID: https://orcid.org/0000-0002-6844-050X Universidade Federal de Sergipe, Brasil

E-mail: ant.sara0023@hotmail.com

Alexrangel Henrique Cruz Santos ORCID: https://orcid.org/0000-0002-3667-5275

Universidade Federal de Sergipe, Brasil

E-mail: alex_rangel2020@ hotmail.com

Amanda Francielly Santos

ORCID: https://orcid.org/0000-0001-7694-4604 Universidade Federal de Sergipe, Brasil

E-mail: francyelly_amanda@hotmail.com

Carolina do Nascimento Alves

ORCID: https://orcid.org/0000-0002-4042-6386

Universidade Federal de Sergipe, Brasil E-mail: carolinaalves270@gmail.com

Thayane Santos Siqueira

ORCID: https://orcid.org/0000-0001-8370-0544 Universidade Federal de Sergipe, Brasil E-mail: Thayane_siqueira@live.com

Ariel Oliveira Celestino

ORCID: https://orcid.org/0000-0003-3907-187X Universidade Federal de Sergipe, Brasil E-mail: biomedica.ariel@gmail.com

Mariana do Rosário Souza

ORCID: https://orcid.org/0000-0002-3139-8483 Universidade Federal de Sergipe, Brasil E-mail:mariana_do_rs@hotmail.com

Beatriz Almeida Santos

ORCID: https://orcid.org/0000-0003-1527-615X Universidade Federal de Sergipe, Brasil

E-mail: Beatriz.enf12@gmail.com

Rosiene Batista Santos

ORCID: https://orcid.org/0000-0001-9584-6874

Universidade Tiradentes, Brasil

E-mail: rosiene_bs@hotmail.com

Luiz André Santos Silva

ORCID: https://orcid.org/0000-0003-1607-8649

Universidade Federal de Sergipe, Brasil

E-mail: luizandressilva@yahoo.com Luana Santos Costa

ORCID: https://orcid.org/0000-0001-9972-6415 Universidade Federal de Sergipe, Brasil

E-mail: luanacosta159@gmail.com

Ana Beatriz da Silva Oliveira

ORCID: https://orcid.org/0000-0002-4512-458X Universidade Federal de Sergipe, Brasil

E-mail: beatrizdso@gmail.com

Magno Pereira Prado

ORCID: https://orcid.org/0000-0003-1848-7219 Universidade Federal de Sergipe, Brasil E-mail: magnoprado@yahoo.com.br 


\title{
Resumo
}

Objetivo: Realizar um levantamento parasitológico de moluscos em São Cristóvão/Sergipe. Métodos: Trata-se de um estudo descritivo e investigativo realizado no município de São Cristóvão. Para tal, Moluscos foram coletados com auxílio de pinça metálica e concha de metal perfurada, colocados em sacos plásticos e encaminhados ao laboratório de Entomologia e Parasitologia Tropical (LEPaT) da Universidade Federal de Sergipe (UFS) para identificação da espécie e do parasitismo. Resultados: Ao total foram coletados 1.669 caramujos, onde desses, 1605 pertenciam ao gênero Biomphalariae, os demais eram Physa sp., Pomacea sp., Lymnea sp. e Melanoide sp. Conclusão: A presença de moluscos do gênero Biomphalariae torna necessário para tornar a manutenção e vida da esquistossomose (barriga d'água) viável, o mesmo, ainda está intimamente ligado a indicadores socioeconômicos precárias, cujo fatores primordiais para prevenção é a educação sanitária e o saneamento básico inerente ao controle do molusco. Portanto, é imprescindível ações profiláticas e trabalhar educação em saúde com a população para melhorar seus hábitos e qualidade de vida.

Palavras-chave: Esquistossomose; Doenças parasitárias; Atenção à saúde; Moluscos.

\begin{abstract}
Objective: To carry out a parasitological survey of molluscs in São Cristóvão/Sergipe. Methods: This is a descriptive and investigative study carried out in the city of São Cristóvão. For this purpose, Molluscs were collected with the aid of metallic tweezers and a perforated metal shell, placed in plastic bags and sent to the Laboratory of Entomology and Tropical Parasitology (LEPaT) of the Federal University of Sergipe (UFS) for identification of the species and the parasitism. Results: A total of 1,669 snails were collected, of which 1605 belonged to the genus Biomphalariae, the others were Physa sp., Pomacea sp., Lymnea sp. and Melanoid sp. Conclusion: The presence of molluscs of the Biomphalariae genus makes it necessary to make the maintenance and life of schistosomiasis (water belly) viable, it is still closely linked to poor socioeconomic indicators, whose primary factors for prevention are health education and sanitation inherent to mollusc control. Therefore, prophylactic actions and working health education with the population are essential to improve their habits and quality of life.
\end{abstract}

Keywords: Schistosomiasis; Parasitic diseases; Delivery of health care; Mollusca.

\section{Resumen}

Objetivo: Realizar un estudio parasitológico de moluscos en São Cristóvão / Sergipe. Métodos: se trata de un estudio descriptivo e investigativo realizado en la ciudad de São Cristóvão. Para ello, se recolectaron moluscos con la ayuda de pinzas metálicas y un caparazón metálico perforado, se colocaron en bolsas plásticas y se enviaron al Laboratorio de Entomología y Parasitología Tropical (LEPaT) de la Universidad Federal de Sergipe (UFS) para la identificación de la especie. y el parasitismo. Resultados: Se recolectaron un total de 1,669 caracoles, de los cuales 1605 pertenecieron al género Biomphalariae, los demás fueron Physa sp., Pomacea sp., Lymnea sp. y Melanoid sp. Conclusión: La presencia de moluscos del género Biomphalariae hace que sea necesario viabilizar el mantenimiento y la vida de la esquistosomiasis (panza de agua), aún se encuentra estrechamente vinculado a indicadores socioeconómicos deficientes, cuyos principales factores de prevención son la educación en salud y el saneamiento inherentes a los moluscos. control. Por tanto, las acciones profilácticas y la educación sanitaria laboral con la población son fundamentales para mejorar sus hábitos y calidad de vida.

Palabras clave: Esquistosomiasis; Enfermedades parasitarias; Atención a la salud; Moluscos.

\section{Introdução}

A esquistossomose é uma doença parasitária, popularmente conhecido por barriga d'água, doença do caramujo, xistose, é ocasionada por platelmintos trematódeos do gênero Schistosoma, e que infecta humanos, no entanto, necessitam, em seu ciclo de vida se desenvolver no interior de caramujos aquáticos pertencentes ao gênero Biomphalaria (Lamberton et al., 2014; Brasil, 2014 A).

A transmissão da esquistossomose acontece através do indivíduo infectado que elimina os ovos através de suas fezes, estes ao entrarem em contato com a água, eclodem e liberam as larvas conhecidas como miracídios, as quais infectam os 
caramujos, depois as larvas abandonam o caramujo na forma de cercarias e ficam livres na água, infectando o indivíduo suscetível através da penetração na pele (Melo, et al., 2016).

Os primeiros relatos no Brasil ocorreram na região Nordeste, com o tempo disseminou-se para as demais regiões acometendo um grande número de pessoas em todo o país levando-as, em muitos casos, a óbito (Brasil, 2014 A). A esquistossomose integra um grupo de doenças negligenciadas segundo a Organização Mundial de Saúde, e constitui um risco para milhões de residentes em áreas endêmicas das diversas regiões do Brasil (Rollemberg, 2011; Vasconcelos et al., 2016 e Albuquerque et al., 2017).

O Ministério da Saúde realizou estudos no período de 2008 a 2016 e evidenciou que o estado de Sergipe um dos maiores índices de prevalência do país, perdendo para Alagoas, Pernambuco e Bahia (ITP, 2019 A). Ainda, o Inquérito Nacional de prevalência (INPEG), em 2018 os estados que apresentaram as maiores proporções de positividade para esquistossomose, nos municípios com até 500 mil habitantes foram: Sergipe (10,67\%), Minas Gerais $(5,81 \%)$, Pernambuco $(3,77 \%)$, Alagoas $(3,35 \%)$, e Bahia $(2,91 \%)$. Nesse Inquérito foram analisados 22 municípios de Sergipe, no entanto o município de São Cristóvão não foi contemplado o inquérito (INPEG, 2018 A).

Segundo estudos o município de São Cristóvão apresenta altos índices endêmicos, com diversos casos de esquistossomose. Diante disso, esse trabalho teve como objetivo realizar um levantamento parasitológico dos moluscos encontrados em São Cristóvão/Sergipe já que, trabalhar no controle dos moluscos do gênero Biomphalaria, educação em saúde com a população e tem sido de grande interesse acadêmico por se tratar do hospedeiro intermediário da esquistossomose (Melo e Coelho et al., 2016).

\section{Metodologia}

\subsection{Tipos de estudo}

Trata-se de um estudo descritivo e investigativo, desenvolvido entre os meses de agosto de 2019 a julho de 2020, realizado no município de São Cristóvão. O município foi a primeira capital do estado e é a quarta cidade mais antiga do país fundada em 1590. Ainda, de acordo com IBGE (2019), o município possui uma população estimada em 90.072 pessoas, sua extensão territorial é de $438,037 \mathrm{~km}^{2}$ entre cidades circunvizinhas e que há vários casos de esquistossomose (Lima et al., 2017).

\subsection{Criadouro}

Foram escolhidos quatro locais do município de São Cristóvão, levando-se em consideração a proximidade com coleções hídricas e a presença de pessoas com esquistossomose. Os locais escolhidos foram: Rua Graciliano F dos Santos Baixa Divinéia; Estrada Rita Cacete - Povoado Colônia Miranda; Chafariz Harasgão Lords - Povoado Colônia Miranda e Rua Valdir Matos - Alta Divinéia. Das localidades mencionadas, o ponto um e quatro são urbanos, já o dois e três são rurais. Assim, foram georreferenciados com auxílio de um receptor GPS (Global Position System), marca Gramin, conforme a tabela abaixo. 
Tabela 1: Bairros e pontos com suas respectivas coordenadas.

\begin{tabular}{|c|c|c|}
\hline LOCAL & PONTO & COORDENADA \\
\hline Rua Graciliano F dos Santos - Baixa & & $11^{\circ} 0{ }^{\prime} 27^{\prime \prime} \mathrm{S}$ \\
\hline Divinéia & 1 & $37^{\circ} 12^{\prime} 42^{\prime \prime} \mathrm{W}$ \\
\hline Estrada Rita Cacete & & $11^{\circ} 0{ }^{\prime} 29^{\prime \prime} \mathrm{S}$ \\
\hline Povoado Colônia Miranda & 2 & $37^{\circ} 12^{\prime} 42^{\prime \prime} \mathrm{W}$ \\
\hline Chafariz HarasgãoLords Povoado & & $11^{\circ} 0{ }^{\prime} 27^{\prime \prime} \mathrm{S}$ \\
\hline Colônia Miranda & 3 & $37^{\circ} 14^{\prime} 23^{\prime \prime} \mathrm{W}$ \\
\hline Rua Valdir Matos & & $11^{\circ} 0,10^{\prime \prime} \mathrm{S}$ \\
\hline Alta Divinéia & 4 & $37^{\circ} 12^{\prime} 39^{\prime \prime} \mathrm{W}$ \\
\hline
\end{tabular}

Fonte: Autoria própria.

\subsection{Coleta de moluscos}

Os moluscos foram coletados com auxílio de pinça metálica e concha de metal perfurada. A técnica de coleta consiste em raspar a concha de captura na vegetação submersa e no fundo dos criadouros e levá-la a superfície, sendo feita a varredura do fundo dos reservatórios hídricos e percorrida a maior área possível (Olivier \& Schneiderman, 1956).

Os caramujos coletados foram encaminhados ao laboratório de Entomologia e Parasitologia Tropical (LEPaT) da Universidade Federal de Sergipe (UFS). No laboratório, os moluscos foram contabilizados, mensurados, colocados em recipientes contendo água sem cloro e cobertos por uma tela para evitar fuga e exposição de insetos.

Foi realizado um teste de positividade para Schistosoma mansoni e outros trematódeos através da exposição dos caramujos à luz. Eles foram colocados em recipientes de vidro, contendo cerca de 3,0 ml de água sem cloro e após isso foram expostos por cerca de uma hora à luz artificial. Após esse tempo, a água do recipiente foi examinada com auxílio de lupa com aumento de 8x para verificar a presença ou não de cercarias. Os caramujos que não liberaram cercarias foram devolvidos aos recipientes e reexpostos à luz semanalmente, num período total de 50 dias (Brasil, 2008 B).

As cercarias obtidas, após a exposição à luz, foram identificadas com o preparo de lâminas, sob microscópio óptico com aumento entre 40 vezes e 100 vezes (Brasil, 2008 Carvalho et al.; 2014). O método de identificação utilizado foram as chaves dicotômicas propostas por (Pinto \& Melo, 2013)

\section{Resultados e Discussão}

Foram coletados 1.669 caramujos ao total, sendo que 1605 pertenciam ao gênero Biomphalariae, após a identificação dos mesmos, os resultados apresentaram que todos eram B. Glabrata.Os demais eram Physa sp., Pomacea sp., Lymnea sp. e Melanoide sp.

O ponto 1 (Rua Graciliano F dos Santos, Baixa Divinéia), apresentou a maior quantidade de Biomphalaria glabrata além da ocorrência de outras espécies, conforme. Os moluscos desse ponto foram coletados dentro de um esgoto doméstico com fluxo de água leve, presença de lixo e vegetação na região, em frente a ele haviam casas e, consequentemente, pessoas em contato. Esse cenário destaca a relevância do peridomicílio na transmissão da doença, já que normalmente a maioria dos focos tem localização peri ou intradomiciliar devido a ação antrópica que leva a degradação do ambiente (Araújo et al., 2007).

Santana (2016), realizou coleta de 1268 caramujos nesse mesmo local e encontrou uma taxa de positividade de 12, 93\%. Isso deixa claro que medidas de controle não têm sido realizada nesse local e além disso, só a presença de caramujos Biomphalaria, já é motivo de preocupação, visto que no local devem existir pessoas com esquistossomose.

No ponto 2 (Estrada Rita Cacete, Povoado Colônia Miranda), apesar de ser um ambiente rural diferentemente do ponto 1 que é urbano, também encontramos esgoto com fluxo leve de água e além da presença de lixo e vegetação ao redor, haviam animais circulando pela área (galinhas, gatos e cachorros principalmente) mas nenhuma pessoa em contato. 
O ponto 3 (Chafariz Harasgão Lords, Povoado Colônia Miranda), foi o mais comprometedor para as pessoas da região visto que este encontrava-se na lateral de casas, no qual havia transição dos próprios moradores. Os caramujos foram encontrados em um córrego com um baixo fluxo de água e que servia de esgoto doméstico, além da presença de vegetação e lixo ao redor, entretanto, após a análise no laboratório nenhuma presença de cercaria foi constatada.

Localidades com alta interferência humana, juntamente com a falta de saneamento básico e a descarga de dejetos domésticos diariamente nesses locais, como este ponto, são consideradas áreas de risco para a transmissão de parasitoses e precisam ser analisadas e solucionadas o quanto antes (Miranda et al; 2016).

O ponto 4 (Rua Valdir Matos, Alta Divinéia), foi marcado pela presença de um minador a céu aberto que, segundo moradores locais, a água é própria para consumo direto). Após a análise dos caramujos, 3,6\% (3) foram positivos. A identificação de criadouros dos moluscos transmissores de esquistossomose ou outros trematódeos em municípios endêmicos, como São Cristóvão, é importante pois possibilita a determinação de áreas com risco de transmissão da doença, fornece subsídios para atividades de controle e vigilância da parasitose, além de auxiliar futuros estudos que serão e devem ser realizados (Nogueira et al, 2016).

Após tais análises, pôde-se concluir a inocorrência de outros trematódeos, até mesmo após a exposição noturna, além de uma baixa taxa de positividade para esquistossomose em relação às amostras coletadas. Tal resultado pode estar relacionado a uma possível baixa taxa de dejetos positivos para esquistossomose sendo lançados em locais próximos ou nos próprios pontos em questão.

Algo semelhante ocorreu no trabalho Miranda onde coletaram 2.585 moluscos e obtiveram uma porcentagem de 0,61\% positivos para S. mansoni. Outro ponto importante a ser destacado é que aérea de coleta desses moluscos foi no ambiente urbano, igual ao presente trabalho. Não foram encontrados caramujos positivos no ambiente rural evidenciando assim que tais patógenos são disseminados mais facilmente em áreas onde o saneamento ambiental é ausente ou deficiente, e onde provavelmente ocorreu maiores impactos ambientais (Miranda et al., 2016).

$\mathrm{O}$ fato de não encontrar caramujos positivos em um local não quer dizer que esse local não precisa ser acompanhado, pelo contrário, pois a qualquer momento esses locais podem se transformar em focos de transmissão. Nos locais onde foram realizadas as coletas, nesse estudo mostram que todas elas apresentavam microflora rica, com muita matéria orgânica, baixa velocidade da água e apresentavam bons lugares para a deposição dos ovos, como plantas, vasilhas de plástico, sacolas, madeiras (Bezerra et al., 2016; Cruz et al., 2020).

\section{Conclusão}

Considerando a prevalência da esquistossomose em Sergipe, no Brasil e no mundo, se faz necessário a divulgação e aplicação de medidas socioeducativas para reduzir os índices de infecção por esquistossomose ou qualquer outra parasitose. Como também, são necessárias medidas consistentes de políticas públicas para a promoção do saneamento básico que envolve rede de abastecimento de água, esgoto, instalação sanitária e tratamento do lixo, sendo primordial para prevenção e controle do molusco que consequentemente implicará em impactos a saúde na região. Sendo assim, esse estudo fornece dados para que outras pesquisas sejam realizadas com o intuito de verificar a prevalência e positividade para esquistossomose no estado de Sergipe, tendo em vista sua endemicidade e presença de caramujos em determinadas regiões.

\section{Referências}

Albuquerque, M. A. C., et al. (2017) Mortality Trends for Neglected Tropical Diseases in the State of Sergipe, Brazil, 1980-2013. Infectious Diseases of Poverty 6 (1), 1-8. https://idpjournal.biomedcentral.com/articles/10.1186/s40249-016-0232-8 
Araújo, K. C. G. M. (2017). Análise espacial dos focos de Biomphalaria glabrata e de casos humanos de esquistossomose mansônica em Porto de Galinhas,

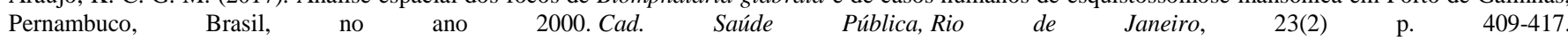
https://www.scielo.br/j/csp/a/x9cgj8bw6fLHN8WZgL4RyJk/abstract/?lang=pt

Bezerra, F. S. M., Fernandez, M. A., \& Thiengo S. C. (2016). Moluscos Transmissores do Schistosoma mansoni no Brasil. A. Parasitologia Humana. (13a ed.), Editora Atheneu, cap.23, 247-256. https://sistema.atenaeditora.com.br/index.php/admin/api/artigoPDF/57602

Brasil. (2014) A. Ministério da Saúde. Secretaria de Vigilância em Saúde. Departamento de Vigilância Epidemiológica. Vigilância da Esquistossomose Mansoni: diretrizes técnicas / Ministério da Saúde, Secretaria de Vigilância em Saúde, Departamento de Vigilância das Doenças Transmissíveis. - 4. ed. Brasília: Ministério da Saúde. https://bvsms.saude.gov.br/bvs/publicacoes/vigilancia_esquistossome_mansoni_diretrizes_tecnicas.pdf

Brasil. (2008) B, Ministério da Saúde. Secretaria de Vigilância em Saúde. Guia de vigilância epidemiológica. 6 ${ }^{\text {a }}$ ed. Brasília: Ministério da Saúde, 1-26. https://bvsms.saude.gov.br/bvs/publicacoes/Guia_Vig_Epid_novo2.pdf

Carvalho, O.S et al. (2014). Moluscos Brasileiros de Importância Médica. Belo Horizonte: Centro de Pesquisas René Rachou/FIOCRUZ,. 92 p. (Série Esquistossomose $\mathrm{n}^{\circ}$ 16). http://pide.cpqrr.fiocruz.br/arquivos/Livro_MoluscosBrasileiros2014.pdf

CDC. Centers for DiseaseControlandPrevention, Departmentof Health \&Human Services,U.S. Schistosomiasis. $<$ https://www.cdc.gov/parasites/schistosomiasis>.

Cruz, J. I. N, Salazar, G. O, \& Corte, R. L. (2020). Retrocesso do Programa de Controle da Esquistossomose no estado de maior prevalência da doença no Brasil. Revista Pan-Amazônica de Saúde, 11, e202000567. https://dx.doi.org/10.5123/s2176-6223202000567

Coelho, P. M. Z; \& Caldeira, R. L. (2016). Critical analysis of molluscicide application in Schistosomiasis control programs in Brazil. InfectDisPoverty.5(1):57, 1-6. https://idpjournal.biomedcentral.com/articles/10.1186/s40249-016-0153-6

IBGE. A Instituto Brasileiro de Geografia e Estatística. São Cristóvão. https://www.ibge.gov.br/cidades-e-estados/se/sao-cristovao.html. 2020. https://www.ibge.gov.br/cidades-e-estados/se/sao-cristovao.html

ITP, Instituto de Tecnologia e Pesquisa. Aperfeiçoamento do medicamento que trata a esquistossomose é feito por pesquisadores do ITP. https://www.itp.org.br/leitura/141

Lamberton, P. H. L. et al. (2014) Sensitivity and Specificity of Multiple Kato-Katz ThickSmearsand a Circulating Cathodic Antigen Test for Schistosoma mansoni DiagnosisPre- and Post-repeated-PraziquantelTreatment. PLOS Neglected Tropical Diseases 8 (9) 3139. https://journals.plos.org/plosntds/article?id=10.1371/journal.pntd.0003139

Lima, V. F. S. et al. (2018). Caracterização da Esquistossomose Mansônica e seus vetores em áreas de foco no Estado de Sergipe, Nordeste do Brasil. Revista Brasileira de Geografia Médica e da Saúde. 14 (27); 30-40. https://redib.org/Record/oai_articulo1452701-caracteriza\%C3\%A7\%C3\%A3o-daesquitossomose-mans\%C3\%B4nica-e-seus-vetores-em-\%C3\%A1 reas-de-foco-estado-de-sergipe-nordeste-do-brasil

Melo, M. M. et al. (2016) Características epidemiológicas da esquistossomose em Pernambuco no período 2000 a 2012. Ciências biológicas e da saúde Recife. 2(3) p. 11-24. https://periodicos.set.edu.br/facipesaude/article/view/3170/2077

Miranda, G. S. et al. (2016). Moluscos límnicos como hospedeiros de trematódeos digenéticos de uma região metropolitana da ilha do Maranhão, Brasil. Revista Scientia Plena.12 (9). https://www.scientiaplena.org.br/sp/article/view/3168

Nogueira, R. A. et al. (2020). Distribuição dos moluscos transmissores da esquistossomose no município endêmico de São Bento, Maranhão, Brasil. RevPatolTrop, 45(1), 10.5216/rpt.v45i3.43508. https://docs.bvsalud.org/biblioref/2018/09/913278/43508-183059-1-pb.pdf

Pinto, H. A; \& Melo, A.L. (2013). Larvas de trematódeos em moluscos do Brasil: panorama e perspectivas após um século de estudos. RevPatolTrop. 42 (4). 369-386. https://www.revistas.ufg.br/iptsp/article/view/27922

Rollemberg, C. V. V et al. (2011). Aspectos epidemiológicos e distribuição geográfica da esquistossomose e geo-helmintos, no Estado de Sergipe, de acordo com os dados do Programa de Controle da Esquistossomose. Rev. Soc. Bras. Med. Trop., Uberaba. 44 (1), 91-96. https://www.scielo.br/j/rsbmt/a/hB5TC57sJKxQbbTZsgbqJpw/abstract/?lang=pt

Santana, K. W. C. (2016.) Dinâmica populacional de moluscos Biomphalaria em potenciais focos de transmissão da esquistossomose em áreas rurais eurbanas do município de São Cristóvão/Se. 2016. 46. Dissertação (Mestrado em Saúde e Ambiente). https://www.scielo.br/j/rbepid/a/vChBWcGXXCmRXJFpy4Zz6wr/?format=pdf\&lang=pt

Santos, D. V. V et al. (2015). Detecção de atividade de fosfatase ácida nos hemócitos de Biomphalaria glabrata(Gastropoda: Planorbidae): um estudo em moluscos da Região Amazônica, Brasil. RevPan-AmazSaude, Ananindeua. 6(1), 45-50. http://scielo.iec.gov.br/scielo.php?script=sci_abstract\&pid=S2176$62232015000100006 \& \operatorname{lng}=$ pt\&nrm=iso

Vasconcelos, R. S. Kovaleski, D. F; \& Junior, Z. C. T. (2016). Doenças Negligenciadas: Revisão da literatura sobre as intervenções Saúde \& Transformação Social 6(2), 114-131. http://incubadora.periodicos.ufsc.br/index.php/saudeetransformacao/article/view/3714/4477 